\title{
Research on the multichannel measurement system in the forest soil respiration
}

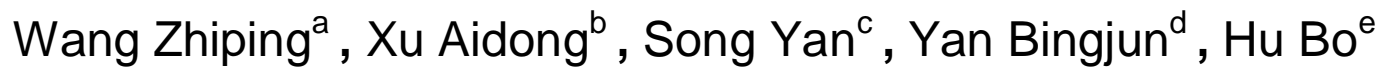 \\ and Liu Qinning ${ }^{\dagger}$ \\ Shenyang Institute of Automation, \\ Chinese Academy of Sciences \\ Shenyang 110016, China \\ awangzhiping@sia.cn, bxad@sia.cn, csongyan@sia.cn, dyanbingjun@sia.cn, ehubo@sia.cn, \\ fliuqinning@sia.cn
}

\begin{abstract}
Keywords: forest soil respiration, multichannel measurement, the least square method
Abstract. According to the requirements of the forest soil respiration experiment, the multichannel measurement system in the forest soil respiration is researched and designed. The system selects the Programmable Logic Controller (PLC) as the core controller, changes the measurement channel by switching the measurement channel unit, analyses the soil respiration gas by the gas analyzer, and saves the soil respiration data by the data collector. The data analysis software is designed according to the requirements of system analysis. The change rate of soil respiration is calculated by the least square method in order to analysis scientifically in forest soil respiration.
\end{abstract}

\section{Introduction}

Forest soil respiration is a hot issue in the field of carbon cycle as an important component of the global carbon cycle. Forest soil respiration directly relates to emit greenhouse gases and affect the world environment safety, and gets more and more attention of governments and scholars. Forest soil respiration in the strict sense refers to all the metabolic effects of undisturbed soil (including the decomposition of the soil organic matter, soil microbial respiration, plant root respiration and soil invertebrate breath) and chemical oxidation of minerals containing carbon[1-2].

Forest soil respiration concentration is usually based on the concentration of $\mathrm{CO} 2$ released from the soil surface, and the concentration measurement methods include indirect measurement and direct measurement. Indirect measurement algorithm is calculated by measuring the concentration of soil respiration related indexes. Direct measurement methods contain static chamber method and dynamic chamber method. Static chamber method accumulates a certain time, and then measures the concentration. Dynamic chamber method directly measures the $\mathrm{CO} 2$ concentration of the experimental boxes, which is currently the most ideal. The advantage of dynamic chamber method can maintain the environmental conditions and the measurement result is more close to the real value [3-4].

The forest soil respiration of experiment is an important research in the carbon cycle field. The experiment generally requires the multi-point measurement in the experimental region and a lot of gas analyzer and data collector. It not only increases the experimental cost, but also leads to the messy test site. Many collecting data are not easy to process, and they bring serious trouble for the scientific workers.

\section{Design of the multichannel measurement system in the forest soil respiration}

The paper takes the forest soil respiration as the research object and designs the multichannel measurement system in the forest soil respiration according to the disadvantage of forest soil respiration experiment. The multichannel measurement system makes use of the programmable logic controller as the core controller and switches the measurement channels by the solenoid valves and relays, and selects the gas analyzer and data collector to collect and save data.

The multichannel measurement system is made up of the switching measurement channel unit, the processor unit and the collection and storage data unit (as shown in Figure 1). The input channel of 
each experiment box respectively connects the collection and storage data unit by the switching measurement channel unit. The processor unit controls the channel to switch by the switching measurement channel unit. The collection and storage data unit analyses the respiration gas of various experimental box, and records the analytical data for processing data.

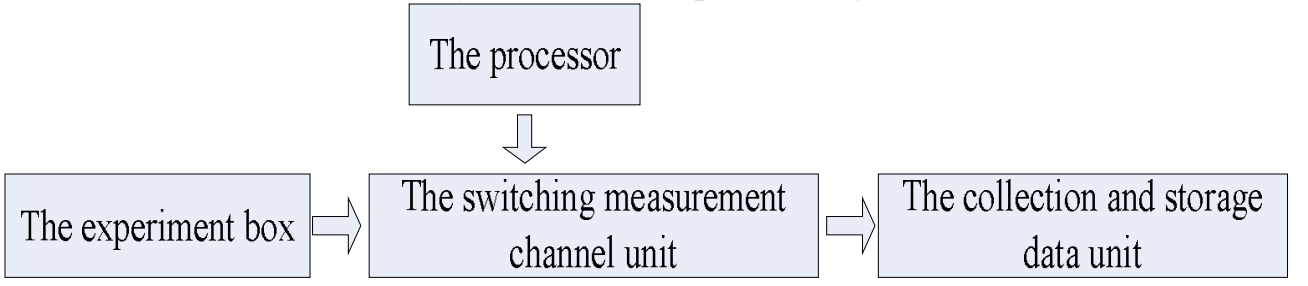

Fig.1 The multichannel measurement diagram

\section{A. The switching measurement channel unit}

The measurement experiment of forest soil respiration often measures the multi-channel signal, but the real-time and synchronization of experiment data is not high. So the experiment can use automatic cycle collection technology and make each experiment box connect the gas analyzer by the switching measurement channel unit, and it not only reduces the experimental cost, but also easily manages to the various experimental boxes [5].

The switching measurement channel unit controls the various experimental boxes to connect the gas analyzer by the solenoid valves, and switches the experiment boxes. So the switching measurement channel unit can switch the measurement channel. In order to research the soil respiration, the experiment needs to make experimental box by transparent organic glass, isolates the soil respiration with the atmosphere, and reduces the effect of atmospheric air in experimental box gas.

The switching measurement channel unit makes the input channel of each experiment box connect respectively the input end of the solenoid valve, and the output end of the solenoid valve connects the gas analyzer by the multi-port pipe. The respiration gas of experimental boxes connects to the input end of the gas analyzer by the mini pump. The gas returning connection mode of experimental box is the same as the input connection mode, but the return gas direction of experimental box acts in the opposite direction to the path of the return gas direction. The diagram of the switching measurement channel unit is shown in figure 2 .

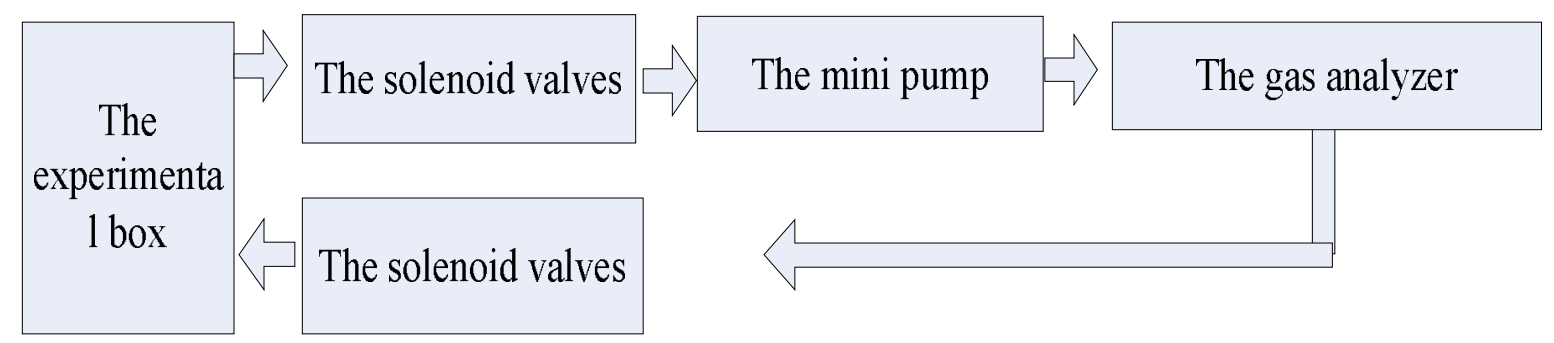

Fig.2 The switching measurement channel unit diagram

The oxygen content of experimental box inside is fixed value, and the oxygen content is decreased after a period of time, so the experimental box need to exchange the gas after working certain time. The switching measurement channel unit makes use of the linear motor to control the experimental box switching. The base of linear motor is fixed by the bracket, and the other end of linear motor is fixed by the cover of experimental box. The linear motor controls the cover of experimental box switching. The cover of experimental box is closed to isolate the soil respiration with the atmosphere, and the cover is open to increase the oxygen content of the experimental box.

\section{B. The processor unit}

The processor unit selects the Programmable Logic Controller (PLC) as the core controller, and makes use of touch screen to set the parameter. The parameter of processor unit includes the system working cycle and experimental box working cycle. The system working cycle is that all enable experimental boxes cost the time. The experimental box working cycle is that each experimental boxes cost the time. 
The processor unit controls each experiment box according to set parameters. The processor unit closes the corresponding experimental box with linear motor, and opens the corresponding solenoid valve, and start the mini pump. The processor unit pumps the gas form the experiment box to the gas analyzer, then waits for reaching the experimental box working cycle. The processor unit judges whether the system working time arrives at the system working cycle. If the working time does not reach at the system working cycle, the processor unit judges whether all enable experimental boxes have worked. If there is the no-working experimental box, the processor unit will close the next enable experimental box. If all enable experimental box have worked and but the system working cycle do not reach, the processor unit shuts down all the solenoid valves and the min pump. The data of the gas analyzer is invalid. The processor unit waits for reaching experimental box working cycle. If the working time reaches system working cycle, the processor unit enters the next cycle.

\section{The collection and storage data unit}

The collection and storage data unit consists of the gas analyzer and data collector. The gas analyzer analyses the $\mathrm{CO} 2 / \mathrm{H} 2 \mathrm{O}$ of experimental box, and the data collector records the gas data and the status of experimental box. Data are identified the number of experimental box according to the recording status of experimental box.

The gas analyzer collects continuously the $\mathrm{CO} 2$ and $\mathrm{H} 2 \mathrm{O}$ concentration of various environmental conditions according to infrared testing technique, and it is used in the fields of soil carbon flux, atmospheric detection, meteorology and botany.

The data collector consists of the measurement module and storage module and provides the function of the sensor measurement, data storage and time setting. The data collector is widely used in fields of agricultural research and industrial testing product.

\section{Design of the processing data software}

The processing data software is developed by Visual Basic according to the requirement of the multichannel measurement system in the forest soil respiration. The software consists of the data classification, data calculation and curve drawing. The data of experimental box are respectively calculated the change rate of $\mathrm{CO} 2$ and $\mathrm{H} 2 \mathrm{O}$ concentration, and curve drew the curve by the least square method.

\section{A. Classify the data}

The forest soil respiration of experiment generally runs a long time in the wild. The data collector saves many data of various experimental boxes (as shown in Figure 3). Figure 3 is an example as 4 experimental boxes. The data in the data collector are identified the number of experimental box according to the recording status of experimental box. The software makes the data of same number experimental box into a file in time sequence. The file is named as the experimental box number, and it is convenient for scientists to query and analysis.

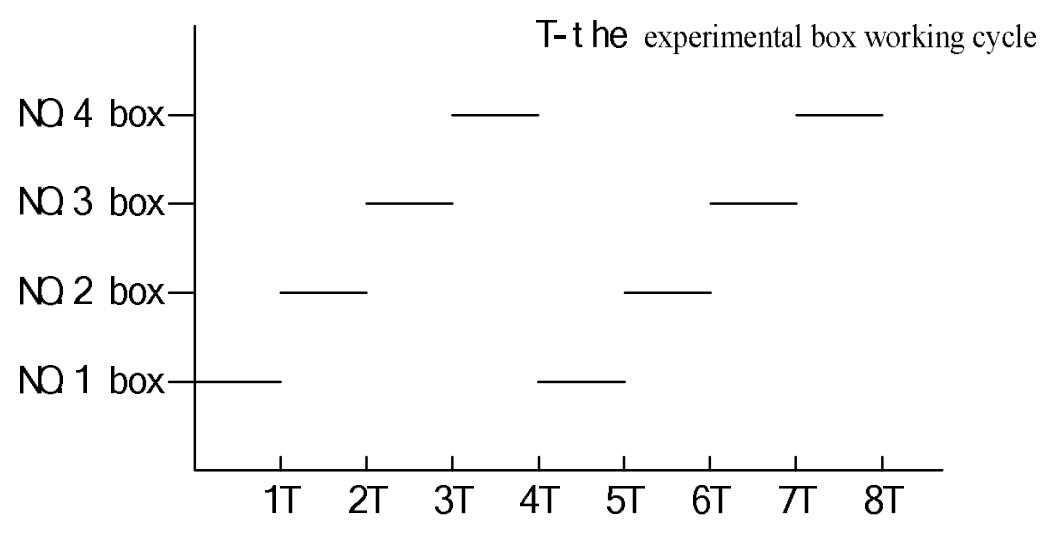

Fig. 3 The experiment time diagram 


\section{B. Calculate the data and draw the curve}

The processing data software gets the $\mathrm{CO} 2 / \mathrm{H} 2 \mathrm{O}$ concentration of each experimental box by the data classification. The software works on the experimental box working cycle as a unit, and calculates the change rate of $\mathrm{CO} 2 / \mathrm{H} 2 \mathrm{O}$ soil respiration and draws the data curve. The error of recording data is big when the switching measurement channel unit switches the experimental box. So the software removes the data when the experimental box is opening or closing.

The software calculates the $\mathrm{CO} 2 / \mathrm{H} 2 \mathrm{O}$ change rate of experimental box working cycle by the least square method and fits the curve.

$\mathrm{X}$ - the $\mathrm{CO} 2 / \mathrm{H} 2 \mathrm{O}$ concentration.

$$
a=\frac{n \sum_{k=0}^{n-1} x_{k} y_{k}-\sum_{k=0}^{n-1} x_{k} \sum_{k=0}^{n-1} y_{k}}{n \sum_{k=0}^{n-1} x_{k}^{2}-\sum_{k=0}^{n-1} x_{k} \sum_{k=0}^{n-1} x_{k}}
$$

$\mathrm{Y}$ - time interval from the beginning experiment to the collection time.

a - the $\mathrm{CO} 2 / \mathrm{H} 2 \mathrm{O}$ change rate.

The software draws the curve of experimental box working cycle by minimizing the error square, and matches the best function data. The $\mathrm{CO} 2 / \mathrm{H} 2 \mathrm{O}$ data change rate and the experiment start time/end time and the experimental box number are together saved to the database file. It is convenient for scientists to unify management [5].

\section{Conclusion}

The paper takes the forest soil respiration as the research object and designs the multichannel measurement system in the forest soil. The system selects the Programmable Logic Controller (PLC) as the core controller, and successfully applies to national field research station of mount baekdu forest ecosystem. The measurement system can collect multi channel data by the switching measurement channel unit and the collection and storage data unit, and calculate the $\mathrm{CO} 2 / \mathrm{H} 2 \mathrm{O}$ change rate by the processing data software. The calculating data are saved in the database file. The multichannel measurement system in the forest soil is convenient for scientists to unify management, which brings great convenience.

\section{Acknowledgment}

This work is supported by the National High Technology Research and Development Program (863 Program), under contract 2013AA040301.

\section{References}

[1] Zhao Ningwei,Gao Chunhua,Li Jianhua.Review on Research Advances and Determination Methods of Soil Respiration.Journal of Shanxi Agricultural Sciences,vol.39,no.1,pp.91-94,2011..

[2] Oechel WC, Vourlitis GL, Hastings SJ, et al . Acclimation ecosystem CO2 exchange in the Alaskan Arctic in response decadal climate warming[J] . Nature, 2000, 406: 978- 981.

[3] Ruan Junwei,Xiang Chenghua,Luo Zongshi,Gong Yuanbo.Advance in Research on Forest Soil Respiration.Chinese Journal of Applied Ecology,vol.17,no.12,pp.2451-2456,2012.

[4] Wang Qian,Liu Hongxing.Design of Switching Devices for Multiple-channel Grouping Input.Computer Measurement and Control,vol.17,no.6,pp.1172-1174,2009.

[5] Jia Xiaoyong,Xu Chuansheng,Bai Xin.The Invention and Way of Thinking on Least Squares.Journal of Northwest University, vol.36,no.3,pp.507-511,2006 